\title{
Preparing Next Generation Graduates for a Global Engineering Workforce:Insights from Tomorrow's Engineers
}

\author{
Ashok K Agrawal ${ }^{1}$, Stephanie Harrington-Hurd ${ }^{2}$ \\ ${ }^{1,2}$ American Society for Engineering Education, Washington, DC \\ ${ }^{1}$ A.Agrawal@asee.org \\ ${ }^{2}$ S.harrington-hurd@ asee.org
}

\begin{abstract}
Transforming Undergraduate Engineering Education (TUEE) project was launched to produce a clear understanding of the qualities engineering graduates should possess and to promote changes in curricula, pedagogy, and academic culture needed to instill those qualities to meet the needs of industry in the $21^{\text {st }}$ century.
\end{abstract}

For the initial phase of this multi-year study, ASEE hosted a two-day workshop Integrating Industry Perspectives designed to hear the "voice of the primary customer employers," in an intensive exploration of the knowledge, skills, and abilities (KSAs) needed in engineering today and in the coming years. The 34 industry attendees identified 36 KSAs that form the core competencies, an array of skills and professional qualities that will help students succeed in a dynamic, rapidly changing field. Industry seeksa T-shaped engineering graduate who brings broad knowledge across domains and the ability to collaborate within a diverse workforce as well as deep expertise within a single domain.

The second workshop Insights from Tomorrow's Engineers was intended to erect a framework for transforming the undergraduate engineering experience. With help from engineering deans, ASEE invited a broadly diverse group of 41 undergraduate and graduate students to ponder 36 characteristics of engineering graduates most sought by industry. The students then joined a two-day workshop in

\footnotetext{
Ashok K Agrawal ${ }^{1}$

${ }^{1}$ American Society for Engineering Education, Washington, DC

${ }^{1}$ A.Agrawal@asee.org
}

\begin{abstract}
Washington, DC to brainstorm ways in which engineering instruction could be improved to meet demands of the contemporary workplace. Overwhelmingly, students concluded that schools were paying insufficient attention to an array of knowledge, skills, and abilities (KSAs) needed to produce the desired T-shaped professional - one who brings broad knowledge across domains, deep expertise within a single domain, and the ability to collaborate with others in a diverse workforce.
\end{abstract}

Keywords: Curriculum transformation, Student voices, Teamwork, Critical thinking, Problem-solving, System thinking and System integration

With support from the National Science Foundation (NSF), the American Society for Engineering Education (ASEE) has launched a series of meetings to develop a new strategy for undergraduate engineering education that meets the needs of industry in the 21st century. Transforming Undergraduate Engineering Education(TUEE) aims to produce a clear understanding of the qualities engineering graduates should possess and to promote changes in curricula, pedagogy, and academic culture needed to instill those qualities in the coming generation of engineers.

\section{Synthesizing and Integrating Industry Perspectives Workshop}

For the initial phase for the TUEE project, "Synthesizing and Integrating Industry Perspectives," ASEE hosted a two-day workshop designed to hear the "voice of the primary customer - employers," from34 invited representatives of companies with an important stake in 
training the future engineering workforce. While the industry representatives were asked to identify the knowledge, skills, and abilities (KSAs) they will demand of engineers in coming years, seven engineering academics were invited to offer ideas on how engineering curricula should be altered to meet employers' needs. The 34 representatives of industry, four staffers and officials from the U.S. intelligence community, and eight academics identified core competencies that remain key, but added an array of skills and professional qualities that will help students succeed in a dynamic, rapidly changing field. They seek a T-shaped engineering graduate who brings broad knowledge across domains and the ability to collaborate within a diverse workforce as well as deep expertise within a single domain.

Prior to the meeting, participants from industry and the seven academics completed an ASEE survey on what they consider the most important engineering KSAs for today and 10 years from now, and the perceived quality of preparation in these areas shown by today's graduates. Questions were drawn from The Engineer of 2020, the latest ABET accreditation criteria, and ASEE conference papers on Boeing-university relations and attributes of the global engineer.

Responses depict a profession under pressure from several directions, with current training unable to meet certain existing industry needs and badly out of sync with the requirements expected in 2023. For instance, they show today's students to be very weak in having an international and global perspective, something of middling importance now but the single most important knowledge area in 10 years' time. Likewise, students' weak foreign language skills, while a minor drawback now, could be a serious impediment in the future.

The survey found today's students coming up short in economics and business, project management, stages of product development, and system integration - all areas of growing importance. Students also fail in meeting expectations in several skills accorded growing importance. These include leadership, decision-making, communication, and the ability to synthesize engineering, business, and societal priorities. At the same time, respondents think students are being well trained in physical and life sciences and statistics, math, and information technology. Indeed, their skills outstrip the importance industry attaches to these fields. Strikingly, strength in math is seen as becoming less important a decade hence than today, as is the ability to apply math and science knowledge and Internet and digital competency, areas where today's students perform well.

\section{Insights from Tomorrow's Engineers Workshop}

"Insights from Tomorrow's Engineers" was the second in a multi-year series of meetings intended to erect a framework for transforming the undergraduate engineering experience. With help from engineering deans, ASEE invited a broadly diverse group of 41 undergraduate and graduate students to ponder 36 characteristics of engineering graduates most sought by industry. The students then joined a two-day workshop in Washington, DC to brainstorm ways in which engineering instruction could be improved to meet demands of the contemporary workplace.

The thirty-six KSAs defined in Phase I by industry were presented to engineering students in the pre-workshop survey. Students were asked to rank the importance of each of the KSAs to the engineering profession as they perceived it. In addition, they were asked to rank the importance of each KSA as it is currently conveyed to them by their institution, as well as the quality of education they are currently receiving in each respective KSA area.

The reported results for each of the 36 KSAs are listed in Table 1, divided into three sections consisting of $12 \mathrm{KSAs}$ each. The table shows many areas in which students as well as academia and the curriculum are closely aligned in their perception of importance. It also highlights areas of misalignment, where students and academia perceive the importance of certain KSAs differently. More importantly, Table 1 indicates which areas of engineering education lack quality and where updates and improvements may be needed.

Table 2 cross-tabulates results from the student pre-meeting survey with data gathered form industry in Phase 1, juxtaposing the importance of each KSA for the engineering profession as perceived by students and industry not only in the present, but also by industry in the next decade. Overall, the comparative data in the table shows a tendency for students to be more closely aligned with what industry perceives will be important in the next decade, compared to industry's priorities today. 
Table 1. Students' perception of KSA's importance for the engienering profession and quality of education received in each area

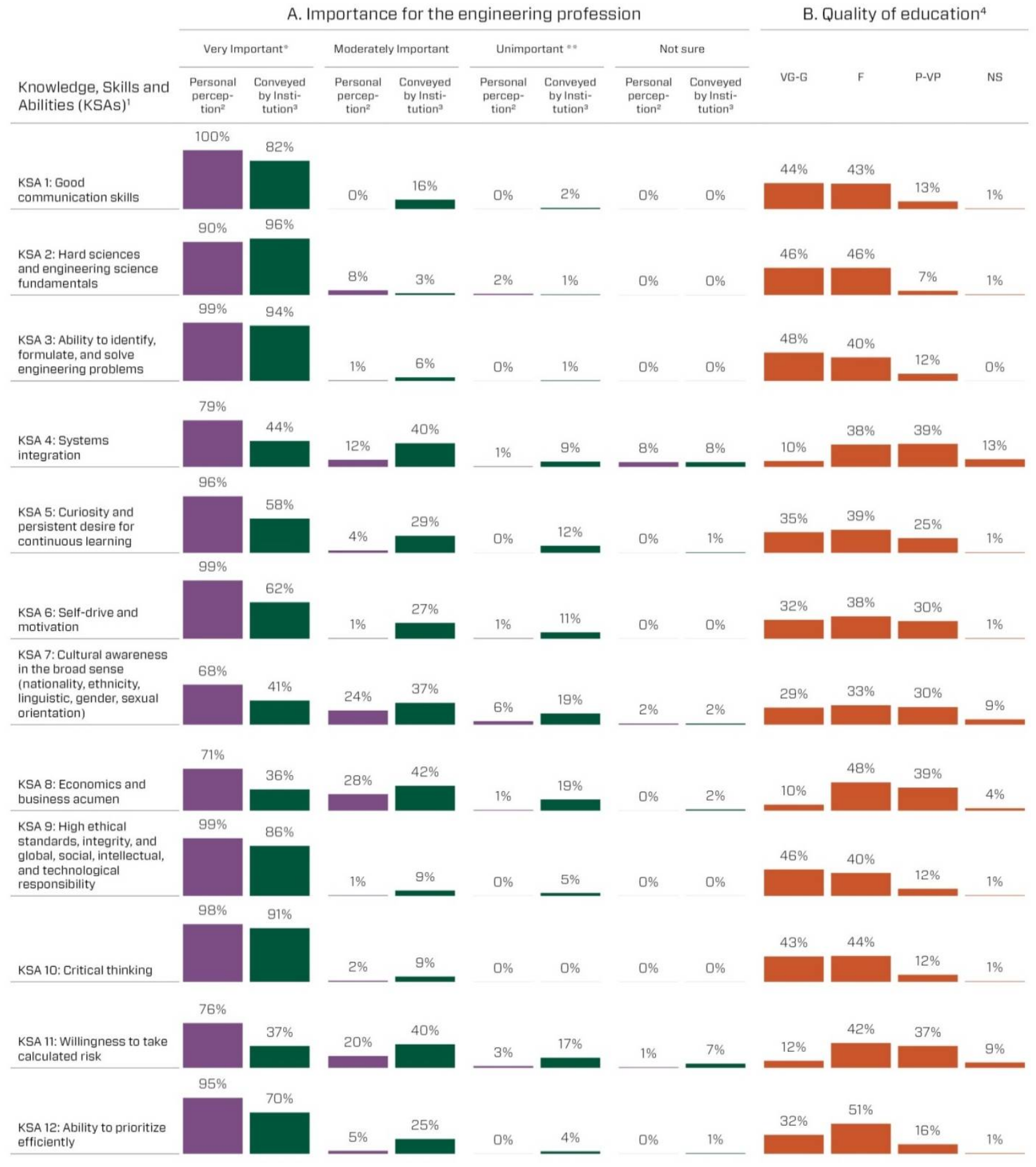

Note. $\mathrm{N}=141$

'KSAs are ordered by priority as initially defined by industry representatives at the TUEE Phase I Workshop,

see report at http://Www.asee.org/TUEE_Phasel_WorkshopReport.pdf

${ }^{2}$ Level of importance as perceived by students.

${ }^{3}$ Level of importance communicated to students through orientation, advising, classes and other activities at their institutions.

${ }^{4}$ Quality of curricular and extra-curricular activities to help develop each area.

VG-G = very good/good; F = fair; P-VP = poor/very poor; NS = Not Sure

"Combines Extremely Important and Important. "Combines Relatively Unimportant and Completely Unimportant 
Table 1. Students' perception of KSA's importance for the engienering profession and quality of education received in each area (continued)

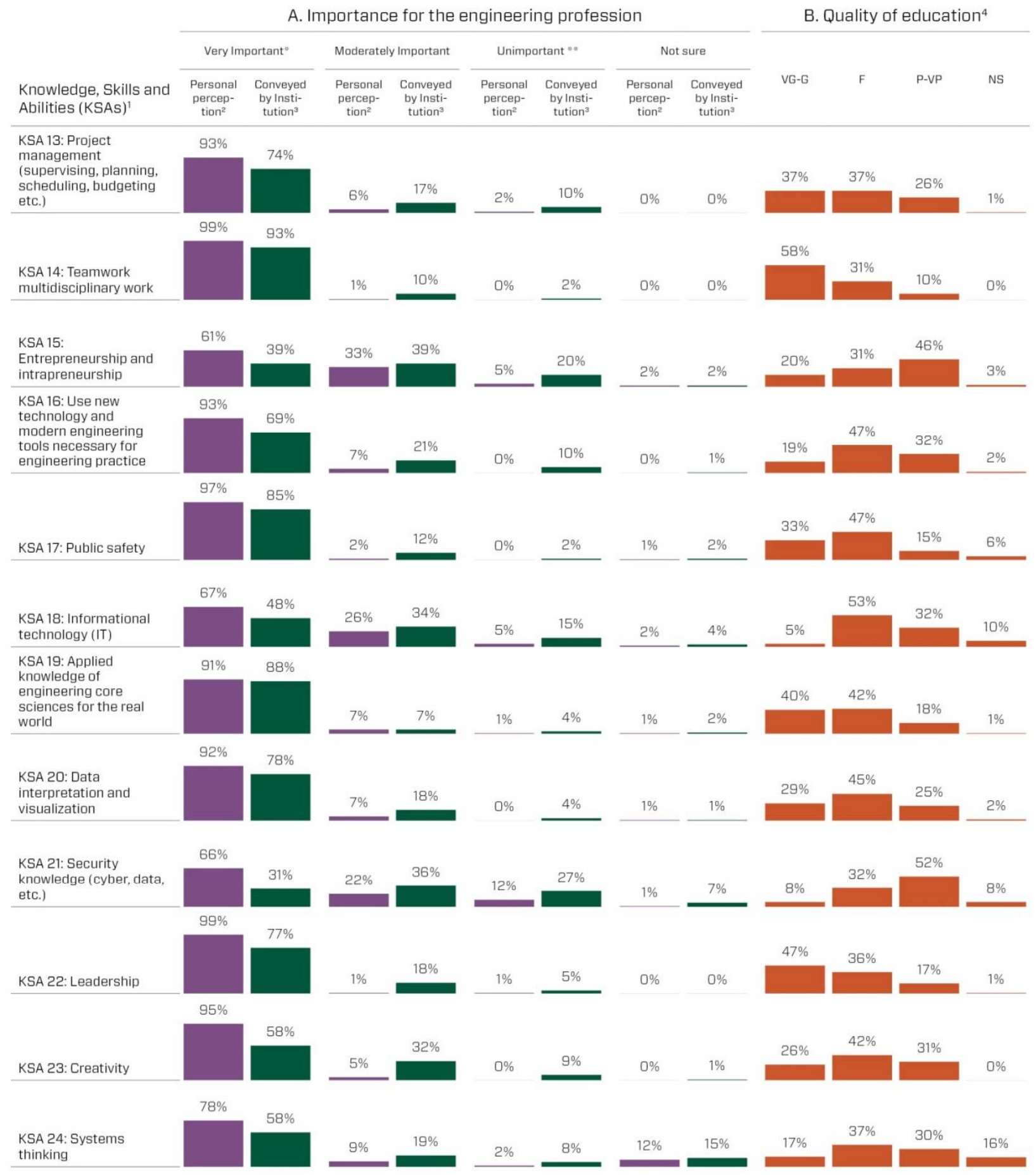

Note. $\mathrm{N}=141$

'KSAs are ordered by priority as initially defined by industry representatives at the TUEE Phase I Workshop,

see report at http://www.asee.org/TUEE_Phasel_WorkshopReport.pdf

${ }^{2}$ Level of importance as perceived by students.

${ }^{3}$ Level of importance communicated to students through orientation, advising, classes and other activities at their institutions.

${ }^{4}$ Quality of curricular and extra-curricular activities to help develop each area

VG-G = very good/good; $F=$ fair; $P$-VP = poor/very poor; NS = Not Sure

"Combines Extremely Important and Important. "Combines Relatively Unimportant and Completely Unimportant 
Table 1. Students' perception of KSA's importance for the engienering profession and quality of education received in each area (continued)

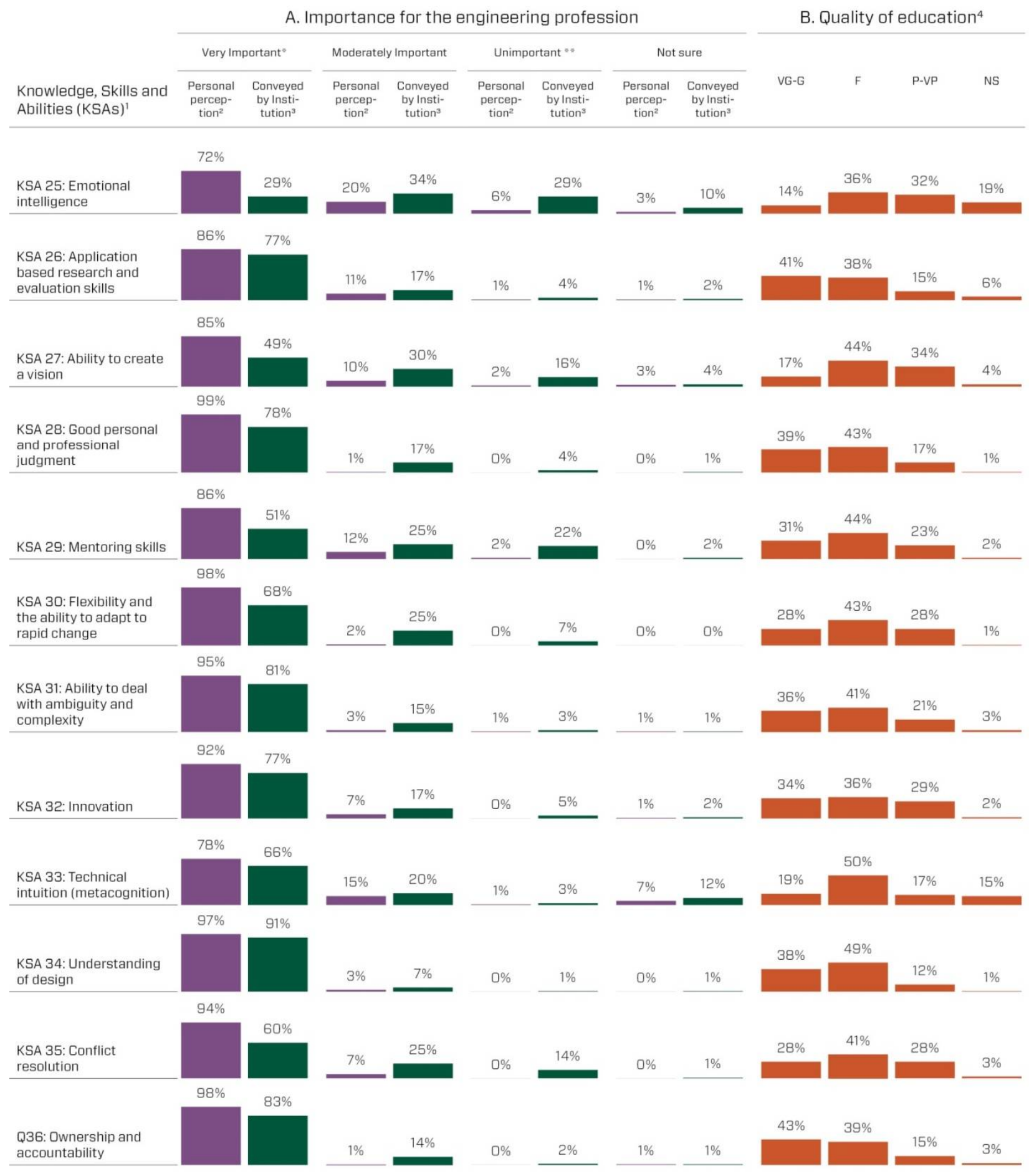

Note. $\mathrm{N}=141$

'KSAs are ordered by priority as initially defined by industry representatives at the TUEE Phase I Workshop,

see report at http://www.asee.org/TUEE_Phasel_WorkshopReport.pdf

${ }^{2}$ Level of importance as perceived by students.

${ }^{3}$ Level of importance communicated to students through orientation, advising, classes and other activities at their institutions.

${ }^{4}$ Quality of curricular and extra-curricular activities to help develop each area.

VG-G = very good/good; F = fair; P-VP = poor/very poor; NS = Not Sure

"Combines Extremely Important and Important. " Combines Relatively Unimportant and Completely Unimportant 
Table 2. Industry vs. Students: Perceptions of the importance of high priority* KSAs for the engineering profession**

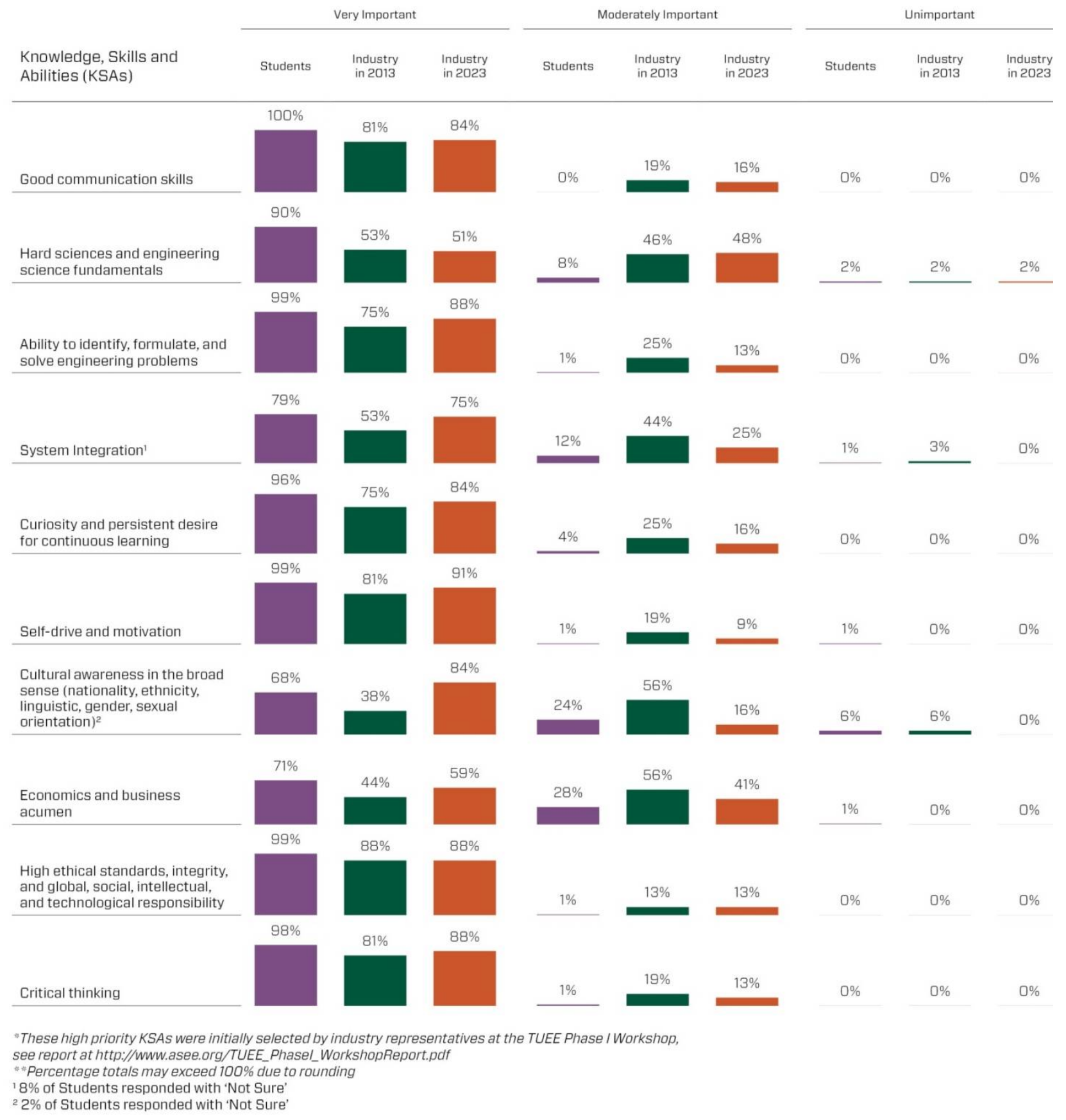




\section{Students' Voices}

A widespread view held among surveyed students revealed that, in their eyes, engineering classes tend to focus largely on the technical aspects of engineering and not so much on how engineers interact in a multidisciplinary and interconnected workforce. While the concrete scientific principles of engineering are absolutely necessary, being able to interact with others and apply knowledge andeducation to multiple areas of life is crucial for the success of the engineering professional. Generally, fundamental engineering and science classes do stress the importance of critical thinking, working in teams, prioritizing, and finding unique way to solve problems. Varying from institutions to institutions, and depending on the individual professors and their backgrounds, the engineering curriculum also often includes coursework and opportunities to build other important professional KSAs such as communications, leadership, and system integration skills, as well as a level of understanding of economics, business and public safety.

According to students, however, no one university teaches the theory of engineering better than another, and it is unlikely that curriculum and theory alone could make a noticeable difference in the quality and preparedness of engineering graduates. In the absence of the 'soft skills' to understand context, identify critical problems, connect the dots, and influence others, theory and technical skill become far less valuable. Ideally, engineers must take classes that will provide them with a holistic education in addition to prolific technical expertise. In the eyes of numerous students, what does make a difference in engineering education is the mix of classwork, practical assignments, and extracurricular activities that prepare students across the board for KSAs. These shape them into members of the workforce and society who bring strong values, a broad perspective, leadership, the ability to communicatewith engineers and non-engineers alike, and quality work and products that tackle real-world problems.

Going beyond hard science and engineering fundamentals in the curriculum, it is important for engineering education to focus on developing the more abstract KSA areas-the soft skills that would help students learn how to apply their education into real life and adapt to engineering workforce situations. According to students, as central as these soft skills are, many are difficult to teach academically. Therefore, it comes down to extracurricular activities, teamwork, and students' own motivation to develop a lot of the softer KSAs.

Project-based learning and opportunities such as design projects, capstones, lab work, research projects, co-ops and internships, membership in professional societies and student organizations, conferences, competitions, and seminars every single year of school build upon the scientific theory. They also bridge technical knowledge with applied skills in industries, society, and the real world, introducing a great variety of necessary skills not covered by the curriculum. They set students up for professional success. Such multidisciplinary teamwork activities combine project-based learning and extracurricular work to develop some of the most important soft skills students will need throughout their engineering program and beyond: leadership, teamwork, communication, time management, prioritization, critical thinking, problem-solving, adaptability, entrepreneurship, self-drive, curiosity, creativity, and risk-taking. Classes that don't have a syllabus, but consist of semester-long student-directed project work without a set schedule of checkpoints could serve as a real incubator for these crucial soft skills.

Design projects and competitions, student design clubs, and capstones were frequently highlighted as prime examples of project-based learning that allowed students to apply their theoretical knowledge in practice and acquire additional vital skills through hands-on engineering work. For instance, one surveyed institution requires their seniors to take a year-long senior engineering design course. This course stresses all of the first $12 \mathrm{KSAs}$ and more. In the course, students work in teams of 4-5 student members to design a product for a local sponsoring company that solves areal-life engineering problem. They work with a faculty advisor and liaison engineers from the sponsoring company throughout the year in product development. Throughout the course, students prepare a proposal, create and follow a project budget, communicate with necessary stakeholders, apply fundamental engineering principles, and inquire about further knowledge necessary to create a solution to the presented engineering problem. Students present finishedproducts at the end of year to the university, sponsoring companies, and the general public in the form of a 20-minute formal presentation, as well as a poster session. Other engineering departments specifically assign design projects at the end of every semester, very much like a senior design course, to help prepare students for engineering tasks, instead of focusing on exams. Furthermore, extracurricular activities such as volunteering with Engineers Without Borders allow students to apply the academic concepts they learn in their classes to projects that have real-world impact. It's an opportunity for aspiring engineers to go through the entire project cycle, from concept-generation to financial management, component design, systems integration, and construction on the ground, while at the same time developing strong communication skills and cultural understanding amongst diverse communities. 
One of the students provided another illustration of the benefits of design projects when he recounted his experience with a Formula SAE car. Almost none of the new members to the Formula team initially had knowledge of what goes into the cars. Because of this, experienced members mentored others to ensure that knowledge was passed down through the team, and that a larger group was available for problem solving. Ultimately, these new members grew into leadership roles during their junior or senior years, which provided exposure to additional lessons, and mentorship and knowledge continuity. Furthermore, with any leadership role there is a degree of accountability, along with the ability to create and lead the design and vision. Students were able to work with one another to apply their preexisting knowledge to the design and fabrication of the car, along with its testing and maintenance. Ultimately, through the mentorship and applied knowledge, students and instructors saw innovation in every car. The team was able to work together through not only the engineering and design challenges, but also through conflict resolution, thus building interpersonal skills and emotional intelligence. As a whole, the experience in Formula SAE provided students not only access to technology and applied engineering knowledge to tackle problems, but also the experience of working with others on a human level.

Overall, many students agreed that freshman and sophomore years of college engineering tend to focus on the fundamental. The much-needed soft skills, context, and practical project and design opportunities only come during the junior and senior years. Students believe that in order to create modern and well-prepared engineers, classes and extracurricular activities should focus on both hard science and soft skills simultaneously from the very beginning and continue throughout the entire degree. At the same time, fundamental scientific concepts and core soft skills should have continuous refreshers so they don't fade away. These could be established and applied in practice. Moreover, applied project design assignments should be attached at the end of each course in engineering school, not just as senior design class in senior year.

Multidisciplinary learning experiences can also be instrumental in teaching students a diverse range of KSAs. The students highlighted a particular multidisciplinary engineering program as an example. The program is running a minor in engineering leadership development where business, education, and engineering majors are able to work together in culturally and professionally diverse teams on projects that teach leadership, business fundamentals (finances, budgets, project proposals, and business plans), technical presentations, ethics, global perspective, cultural awareness, and how they all connect to the field of engineering to solve societal needs. Some schools also require students to take an engineering clinic every semester. The clinic is a class where students work in a team on a multidisciplinary research-based project. This helps cultivate curiosity and a persistent desire for continuous learning, along with self-drive and motivation. During the clinics, students learn a lot about not just economics, but also ethics and integrity by researching and presenting an engineering ethics case. This teaches students about high ethical standards, integrity, and global, social, intellectual, environmental, and technological responsibility.

Extracurricular activities such as involvement in, or leadership of, project management (design, lab, capstones, etc.), student clubs and organizations, student chapters of professional societies, and community work are also highly effective in developing KSAs. These can cultivate strong leadership, teamwork, management and communicationskills, self-motivation, critical thinking, problem-solving, and system thinking and system integration abilities. All of these activities involve working with a number of different stakeholders, ranging from executives to volunteers, full-time staff, administration, and external groups. Extracurricular activities could also be multidisciplinary, providing opportunities to work with peers from other majors.

\section{Conclusions}

Recommendations from the Synthesizing and Integrating Industry Perspectivesworkshop included:

- Industry still values a solid foundation in math and science, although the relative importance of math may diminish slightly in the years ahead.

- Good communication skills, persistence, curious learning capability, drive and motivation, economics and business acumen, high ethical standards, critical thinking, and willingness to take calculated risks are important skills and attributes for all engineering graduates.

- Changes in pedagogical approach and in-and out-of classroom experience will be necessary to instill these characteristics.

- Universities will need to adjust faculty reward structures to place more of a premium on teaching, promote more cross-disciplinary instruction, and welcome involvement by industry in supplying case studies, mentorship of students, and shared laboratory experiences.

- Industry will need to recognize a shared responsibility in developing $\mathrm{T}$-shaped engineers.

- Industry and academe will need to collaborate in creating internships in industry for both students and faculty; in designing authentic learning experiences that occur before traditional capstone projects; and in a shared awareness that barriers between universities and companies serve neither. 
Recommendations that emerged from Insights from Tomorrow's Engineers workshop included:

- Early access to mentoring, engineering experiences, and advising, with an entire community - students, faculty, student organizations, and industry - each playing a role.

- Open-ended, interdisciplinary projects undertaken by groups that change composition over time, forcing students to adapt to new partners.

- A focus on real-world impact, so as to show students why what they're being taught is important. The impact could be illustrated by case studies and reinforced with internships, coops, and guest speakers.

- Enhancing the connection between students and professors, thus creating a sense of community.

- Team design projects starting in freshman year that benefit someone or some organization.

- Building design projects into upper-level courses.

- A diversity of professors' gender, ethnic background, and experience in industry or academe.

- Instead or rotating instructors of required courses, allow faculty members to teach subjects they're passionate about or really skilled at teaching.

- Show the applications to engineering in first-year math and science courses - calculus, physics, and chemistry.

- Make teaching part of the basis for securing tenure. For tenured faculty, evaluate teaching as part of salary reviews.
- Improve accountability by assessing whether courses fulfill the promise suggested in syllabi. For instance, did students reach ABET-level outcomes?

- Encourage faculty to be creative in supplying realworld examples.

- Incorporate writing and presentations in various courses to build students' communication skills.

- Offer minor credit or certificates of proficiency in soft skills.

- Redistribute grading to increase the value of project-based learning as opposed to exams.

\section{Acknowledgements}

This project was performed under a grant (DUE\#1448876) from the National Science Foundation.

I want to express my most sincere thanks to my colleagues Dr. Rocio Chavela and Stephanie HarringtonHurd for their support in designing and supporting this workshop and to Mark Matthews for preparing the workshop report.

\section{References}

American Association for Engineering Education, (2013).Transforming Undergraduate Education in Engineering, Phase I: Synthesizing and Integration Industry Perspectives.

American Association for Engineering Education, (to be published in 2015). Transforming Undergraduate Education in Engineering, Phase II: Insights from Tomorrow's Engineers. 